\title{
Arbor
}

\section{La política europea de seguridad y defensa en el escenario internacional actual}

\section{Esther Barbé, Alfonso González Bondia}

Arbor CLXXII, 678 (Junio 2002), 357-382 pp.

\section{Introducción}

El papel de la Unión Europea en la sociedad internacional actual depende en gran medida de la calidad y eficacia de los instrumentos que tiene a su alcance. En este sentido, las previsiones contenidas en el Tratado de la Unión Europea y en los Tratados constitutivos de las Comunidades Europeas, y que han sido perfeccionadas por los tratados de Amsterdam y de Niza, pretenden dotar a la Unión de los instrumentos en los ámbitos económico, político y militar, necesarios para desarrollar su acción exterior.

Desde esta perspectiva, la política europea de seguridad y defensa se considera un componente fundamental del conjunto de la acción exterior de la Unión Europea. Existe una visión generalizada en torno al hecho de que sin instrumentos militares no es posible esperar una defensa eficaz de los intereses de la Unión Europea en el mundo. A este respecto hay que decir, sin embargo, que existe una opinión discordante con esta idea, aquella que defiende que la auténtica baza de los europeos es justamente su perfil no militar, su calidad de potencia civil (actor destacado en campos como el comercio, la cooperación al desarrollo, la rehabilitación post-conflicto, etc. ${ }^{1}$. Ahí es donde la UE se ha ganado su prestigio en el mundo. Esta observación de partida sirve para recordarnos que estamos frente a una política europea no exenta de polémica.

Este artículo, sin desconocer ese debate fundamental para la definición del papel de la Unión en el mundo, se va a centrar en el análisis del reciente desarrollo de la política europea de seguridad y de defensa. Para 
poder realizar un análisis certero de lo que cabe esperar de la política europea de seguridad y defensa es necesario conocer con exactitud el marco jurídico-institucional que han establecido los Estados miembros a la luz del Tratado de la Unión Europea. A pesar del esfuerzo realizado en los últimos años para establecer unas reglas de juego mínimamente eficaces, que permitan desarrollar una política europea de seguridad y defensa en el marco de la Unión Europea, el diseño jurídico-institucional resultante plantea algunos interrogantes, en forma de desafíos internos y externos, a los que es necesario dar respuesta. Sin ello, será prácticamente imposible que la UE consiga asumir un papel protagonista en la escena internacional actual. El papel de la UE en dicha escena y los instrumentos para actuar en la misma se han convertido en un tema de primer orden para la opinión pública europea. Ahí están Afganistán y el Próximo Oriente para recordárnoslo. Una vez más, el sistema internacional marca la agenda de la UE, incluso en momentos en los que la vida interna parecería marcar la agenda política de la UE (ampliación, Convención).

Así, en las siguientes páginas se procede, de entrada, al estudio de las normas que establecen los limites materiales de la acción de la Unión Europea, así como de la estructura orgánica y de los instrumentos normativos con los que cuenta para desarrollar sus actividades en este ámbito. A continuación, se identifican y analizan tres cuestiones sobre el desarrollo interno de la Unión que, a nuestro juicio, condicionan sobremanera la eficacia de la política europea de seguridad y defensa; a saber, la cohesión de la Unión en este ámbito, la capacidad europea para desarrollar operaciones de gestión de crisis y la independencia técnica e industrial de los europeos en el sector de la defensa. Finalmente, se repasaran tres temas pendientes en el marco de las relaciones exteriores de la Unión Europea que también pueden influir en el devenir de la PESD; a saber, las relaciones con Estados Unidos, la participación de terceros Estados en las actividades de la Unión Europea en este ámbito y las relaciones con la OTAN.

\section{La definición de la PESD}

La celebración del Tratado de la Unión Europea ha permitido avanzar hacia el tratamiento integral de los temas relativos a la seguridad, la cual cosa se ha visto posteriormente confirmada y consolidada con las modificaciones que el Tratado de Amsterdam y el Tratado de Niza han introducido en el TUE ${ }^{2}$. 
En la actualidad, el alcance material de la PESC se encuentra definido en el artículo 17.1 del TUE donde se afirma que «abarcará todas las cuestiones relativas a la seguridad europea", aunque a continuación realiza una aproximación gradual en dos etapas a las cuestiones relativas a la defensa: en la primera se procederá a una definición progresiva de la política de defensa común, mientras que la segunda supondría el eventual establecimiento de «una defensa común». A su vez, se ha previsto que el Consejo Europeo se encargará de decidir el momento en el que se llegue a establecer una defensa común, para lo que será necesaria además la ratificación posterior de los Estados miembros de acuerdo con sus respectivas normas constitucionales.

Sin embargo, la aplicación efectiva de esta previsión ha estado limitada desde el principio por otras disposiciones del propio TUE. En primer lugar, los miembros de la Unión Europea se han comprometido a mantener íntegramente el acervo comunitario, por lo que se debería evitar cualquier intromisión de la PESC en el marco competencial de las Comunidades Europeas. Por otra parte, la política de la Unión no puede contravenir las obligaciones contraídas en el marco de la OTAN por los miembros que consideren que su defensa común se realiza dentro de la organización transatlántica y además ha de ser compatible con la política común de seguridad y de defensa que se establezca en dicho marco. Finalmente, se estableció que la PESC tampoco puede afectar a la singularidad de la política de seguridad y de defensa de determinados Estados miembros, en clara referencia a los países neutrales (Irlanda, Austria, Suecia, Finlandia).

La indefinición inicial del propio tratado y la falta de consenso entre sus Estados miembros ha condicionado sobremanera la delimitación material de lo que la Unión Europea incluye en el marco de la política europea de seguridad y defensa. Por ello, una de las novedades más significativas que se pudo apreciar en la nueva regulación establecida por el Tratado de Amsterdam sobre los aspectos relativos a la seguridad y defensa de la PESC fue sin duda la concreción expresa de algunas de las actividades que se inscriben en el marco de la política de defensa de la Unión Europea, habitualmente conocidas como misiones Petersberg. En este sentido, el artículo 17.2 del TUE establece que se podrá acordar la realización de «misiones humanitarias y de rescate, misiones de mantenimiento de la paz y misiones en las que intervengan fuerzas de combate para la gestión de crisis, incluidas las misiones de restablecimiento de la paz» ${ }^{3}$, mientras que el párrafo final del apartado primero del artículo 17 permite incluir también la cooperación entre los Estados miembros en el sector del armamento. No obstante, conviene señalar que la introducción de estas nuevas previsiones no significa que con la versión anterior del 
TUE no fuese posible la realización de este tipo de actividades, aunque no cabe duda que la nueva redacción supone una clarificación digna de mención, que muestra el nivel de acuerdo alcanzado entre los miembros de la Unión Europea sobre la dimensión de defensa de la $\mathrm{PESC}{ }^{4}$. $\mathrm{Al}$ respecto, el Parlamento Europeo, tras señalar la necesidad de trabajar con conceptos precisos, afirmó que en el marco de la PESD la noción de seguridad tiene carácter global e indivisible y que la seguridad de unos contribuye a la de los demás, mientras que a su juicio la defensa estaba constituida por un conjunto de medios militares al servicio de la seguridad ${ }^{5}$.

El mero análisis de las diversas disposiciones contenidas en el TUE sobre la política de seguridad y defensa permite avanzar que su desarrollo será lento y tortuoso (toma de decisiones, ausencia de cooperación reforzada) ${ }^{6}$. Esta percepción se acrecienta aun más con la posibilidad, ya prevista en la versión original del TUE y que se mantiene en la actualidad, de que dos o más Estados miembros cooperen de manera más intensa, bilateralmente o en el marco de la OTAN o de la UEO, siempre que ello no contravenga u obstaculice la PESC. Esta previsión abre la puerta a que se profundice en determinados ámbitos de manera restringida, a la espera de que en el marco de la Unión Europea se produjese un consenso suficiente que permita incorporarlos a la política de defensa común ${ }^{7}$. Desde esta perspectiva se debe analizar la creación de diferentes brigadas multinacionales, de las que fue pionero el Eurocuerpo, o la constitución en 1996 de la Organización Conjunta de Cooperación en materia de Armamento (OCCAR) ${ }^{8}$.

Aunque desde la entrada en vigor del TUE ya era jurídicamente posible la elaboración por parte de la Unión Europea de una política de defensa común, las discrepancias existentes entre sus miembros, ejemplificada en la divergencia franco-británica, apenas permitieron la adopción de decisiones con repercusiones militares. Así, para que la Unión Europea pudiese realmente desarrollar un papel más activo en este ámbito era necesario superar las diferencias existentes en su seno entre los Estados proatlantistas, los tradicionalmente neutrales y los más europeís$\operatorname{tas}^{9}$. El paso definitivo en este sentido se dio con la Declaración sobre la defensa europea emitida tras la cumbre franco-británica celebrada en Saint-Malo el 4 de diciembre de 1998, donde se reconoció por primera vez la posibilidad de desarrollar una capacidad militar autónoma en el seno de la Unión Europea. Este acuerdo entre los dos miembros más significativos de las posiciones europeístas y proatlantistas ha posibilitado la aprobación de diversas declaraciones e informes en el seno de la Unión Europea que fijan las bases de la política europea común de seguridad y defensa. Hay que apuntar, al mismo tiempo, que el desbloqueo británico 
a la política de seguridad y defensa hacía precisamente de este país un líder natural, sino el líder natural, de dicha política en el marco de la UE. Hasta cierto punto, con esta decisión, el gobierno de Blair perseguía una clara potenciación de su papel en la Unión, sobre todo en momentos en los que se quedaba al margen del avance más importante de la misma (el euro). En otras palabras, todo hacía suponer en 1998 que la militarización de la agenda europea reforzaría el papel de Gran Bretaña. Los hechos del 11 de septiembre, y sus consecuencias posteriores, así lo han confirmado.

En términos institucionales, los cimientos de la futura política europea común de seguridad y defensa se pusieron en la «Declaración del Consejo Europeo sobre el refuerzo de la política europea común de seguridad y de defensa" (Colonia, 3 de junio de 1999), donde se establecieron los criterios básicos que regirán la actuación de la Unión en este ámbito, y que se han visto consolidados y desarrollados en diversos documentos y decisiones adoptadas posteriormente por el Consejo Europeo y el Consejo.

En la Declaración del Consejo Europeo de Colonia se definen dos de los temas que pasan a ser prioritarios en este ámbito. Por una parte, se acuerdan las líneas básicas para dotar a la Unión Europea de los medios y capacidades que le permitan adoptar y ejecutar autónomamente decisiones en todas las tareas de prevención de conflictos y gestión de crisis definidas por el TUE. Si bien, hay que decir que en materia de prevención de conflictos y de gestión de crisis existe una falta total de clarificación en cuanto a alcances, contenidos, etc. Esta es una de las grandes debilidades de la Unión, que ha dado lugar en los últimos años, tanto a documentos de la Unión con voluntad clarificadora ${ }^{10}$ como a una importante literatura al respecto ${ }^{11}$. En segundo lugar, la Declaración de Colonia reconoce la necesidad de fortalecer la base industrial y técnica de la defensa para que sea más competitiva y dinámica.

Por lo que se refiere al primer ámbito de actuación, conviene subrayar que, como ha afirmado el Consejo Europeo, el uso de recursos militares por parte de la Unión Europea no incluye la organización de la defensa colectiva de los Estados miembros, que sigue bajo la responsabilidad de la OTAN. A su vez, el hecho de que la Unión Europea haya reconocido expresamente, no sólo que actuará de conformidad con los principios de la Carta de las Naciones Unidas, sino también que el Consejo de Seguridad es el primer responsable del mantenimiento de la paz y la seguridad internacionales, podría indicar su disposición a evitar acciones militares seriamente cuestionables desde el punto de vista de su legalidad internacional, como algunas de las realizadas por la OTAN durante el con- 
flicto en Kosovo. Aun así, no debe olvidarse que en aquellas operaciones estuvieron directamente involucrados la mayor parte de los miembros de la Unión Europea, por lo que no cabe ser muy optimista sobre el alcance real de este compromiso.

Una de las consecuencias del desarrollo de la PESD ha sido la adopción de nuevos procedimientos y la creación de un conjunto de órganos que han de permitir a la UE preparar y adoptar decisiones en los casos en los que se tenga que enfrentar a la gestión de crisis internacionales ${ }^{12}$. Con este objetivo, se ha acordado la necesidad de convocar periódicamente, y cuando sea necesario, reuniones del Consejo de Asuntos Generales con la presencia de los ministros de Defensa. La voluntad expresada por algunos países, entre ellos España, de contar con reuniones del Consejo a nivel exclusivamente de Ministros de Defensa se encontró con la oposición de otros socios. De cara al desarrollo de sus funciones en materia de gestión de crisis, el Consejo cuenta, desde principios de 2001, con la asistencia de los siguientes órganos: el Comité Político y de Seguridad (COPS), que prepara las decisiones del Consejo y ejerce, bajo la autoridad del Consejo, el control político y la dirección estratégica de las operaciones de gestión de crisis; el Comité militar (CMUE), que asesora en temas militares y elabora recomendaciones para el COPS; el Estado Mayor (EMUE) ${ }^{13}$, que realiza tareas de asistencia eminentemente técnicas; y la Unidad de Planificación Política y Alerta Rápida (UPPAR), que cuenta con un Centro de situación para abastecer de información fidedigna a los órganos de decisión sobre la situación en diferentes puntos del planeta ${ }^{14}$. A todos estos órganos se les ha de sumar también el Centro de Satélites y el Instituto de Estudios sobre la Seguridad que han sido transferidos por la UEO como agencias autónomas de la Unión Europea.

Para la ejecución de las operaciones militares, la Unión Europea pretende seguir el modelo que se había previsto en el marco de la UEO, en virtud del cual se distingue entre las misiones dirigidas por la UE que utilicen medios y recursos de la OTAN y las misiones dirigidas por la UE que usen medios y recursos nacionales o multinacionales europeos previamente identificados. Para la implementación del primer supuesto será necesario el establecimiento de relaciones estables con la OTAN, mientras que para poder hacer efectiva la segunda posibilidad los Estados miembros se han comprometido a mejorar su capacidad militar con el objetivo de que en el año 2003, como muy tarde, se esté en condiciones de desplegar en el plazo de sesenta días y de mantener durante un mínimo de un año fuerzas militares de hasta 60.000 personas capaces de realizar cualquiera de las misiones previstas en el artículo 17.2 del TUE. De he- 
cho dichas Fuerzas de Reacción Rápida, declaradas operativas desde el Consejo Europeo de Niza, han de llevar a cabo sus primeros ejercicios durante la presidencia española. A pesar de las limitaciones de este instrumento militar de la UE, su simple existencia va a permitir evidenciar mejor si el problema de la indecisión europea, que tanto afectó a la imagen de la UE en el caso de Bosnia, se debía tan sólo a la falta de instrumentos comunes o, contrariamente, el auténtico problema ha sido y es la falta de voluntad política para actuar con firmeza en situaciones de conflicto. Dónde, cuándo y por qué actuar con firmeza en el sistema internacional son preguntas fundamentales que la UE aún no ha afrontado, careciendo de una concepción estratégica clara ${ }^{15}$.

El desarrollo de las capacidades militares de la PESD ha ido acompañado, en paralelo, de la creación de instrumentos en el ámbito de la gestión civil de crisis. Una vez más han sido los miembros neutrales y nórdicos de la UE los que han jugado un papel más destacado. Así, la presidencia finlandesa dio un impulso notable al mandato recibido del Consejo Europeo de Colonia para mejorar y utilizar mecanismos de gestión civil de crisis, poniendo especial énfasis en la idea de respuesta rápida y de coordinación en la respuesta (coherencia interpilares), tal y como recogía el Informe presentado al Consejo Europeo de Helsinki, el 10 y 11 de diciembre de 1999. La presidencia sueca, por su parte, ha impulsado el fortalecimiento de la dimensión civil, y durante la misma se creó un Comité para la gestión civil de crisis que asiste al Consejo y un Mecanismo de reacción rápida ${ }^{16}$. Además, se establecieron también los objetivos de capacidades de policía y se acordaron las finalidades de la gestión civil de crisis que incluye las operaciones de policía, el fortalecimiento del Estado de Derecho, el fortalecimiento de la administración civil del Estado y las operaciones de protección civil.

\section{Los desafíos internos}

La Unión Europea se enfrenta a un conjunto de desafíos internos que condicionan de manera importante su papel en la escena internacional. El primero de ellos está relacionado con la cohesión y coherencia de la política europea de seguridad y defensa, el segundo tiene que ver con la disponibilidad de capacidades militares y civiles que permitan a la UE desarrollar operaciones de gestión de crisis, y el tercero se centra en el establecimiento de una base industrial y técnica europea de la defensa que le permitiría a la Unión un abastecimiento autónomo y adecuado a sus necesidades. 
Uno de los retos a los que se ha de enfrentar la Unión Europea es el de la búsqueda de la cohesión política entre sus miembros a la hora de definir la Política Europea de Seguridad y Defensa ${ }^{17}$. Por una parte, la coexistencia de atlantistas, europeístas y neutrales en el seno de la Unión Europea, así como las diversas alianzas estratégicas de algunos miembros en diferentes partes del planeta o las diferentes visiones sobre como enfrentarse a las crisis internacionales son problemas que dificultan la coherencia y desarrollo de una PESD eficaz. Por otra parte, el diseño resultante en el que coexisten la lógica comunitaria y la de cooperación intergubernamental para desarrollar la acción exterior de la Unión Europea, con el consiguiente peligro de incoherencia entre el primer y el segundo pilar, y la ambigüedad con la que se han definido algunas de las funciones de los diferentes órganos e instituciones en el marco de la Política Exterior y de Seguridad Común son dos factores más que se suman a las dificultades que se pueden plantear a la hora de dotar de cierta cohesión a la política europea de seguridad y defensa.

Las disposiciones del TUE muestran claramente el difícil compromiso al que se llegó entre neutrales, europeístas y atlantistas. Por una parte, los limites impuestos a la PESD respecto a la actividad de la OTAN es un ejemplo de lo que se acaba de exponer. Por otra parte, algunos Estados miembros de la Unión Europea pretenden mantener un alto grado de autonomía para poder decidir su participación en el proceso de adopción y ejecución de decisiones con repercusiones en el ámbito de la defensa.

Esta última posibilidad ya había sido garantizada expresamente en el antiguo artículo J.4.4 del TUE, al establecer que se debería respetar el «carácter específico de la política de seguridad y de defensa de determinados Estados miembros». Esta disposición, que se incluyó inicialmente para solucionar la tradicional neutralidad irlandesa, sirvió en aquel momento para resolver el problema derivado de las dificultades planteadas durante el proceso de ratificación danesa del TUE, que se desbloqueó definitivamente con la Declaración de los Jefes de Estado y de gobierno, reunidos en el seno del Consejo Europeo, que reconocía que «Dinamarca no participa en la elaboración y aplicación de las decisiones y acciones de la Unión que tengan repercusiones en el ámbito de la defensa, aunque no impedirá el desarrollo de una cooperación más estrecha entre los Estados miembros en este ámbito» ${ }^{18}$. La situación danesa se ha convertido, de hecho, en el marco de la Europa de los Quince en un fenómeno único, que está provocando malestar entre las mismas fuerzas políticas danesas, tal y como demostró la negociación de Niza ${ }^{19}$.

Así, aunque el nuevo artículo 17.1 párrafo tercero del TUE mantiene la previsión del antiguo artículo J.4.4, cabe señalar que la posición de los 
Estados neutrales ha evolucionado notablemente, como ya hemos visto, respecto a la posibilidad de participar activamente en acciones desarrolladas por organizaciones de cooperación militar. En este sentido, baste recordar que Austria, Finlandia y Suecia, desde su adhesión a la Unión Europea, han participado en todas las operaciones de la UEO, e incluso que Irlanda ha participado en la SFOR, en Bosnia, y la KFOR, en Kosovo. Por este motivo, actualmente es evidente que, aún disponiendo de la posibilidad de mantenerse puntualmente al margen, dichos Estados participan cada vez de manera más activa en la elaboración de la Política Europea de Seguridad y Defensa de la Unión Europea, tal y como ya se ha visto en el mismo momento de reformar el Tratado en relación con las funciones de la PESD.

A pesar de todo, conviene señalar que el precario acuerdo entre los tradicionalmente llamados atlantistas, los europeístas y los neutrales, que ha dado lugar, en primer lugar, a una acuerdo sobre las disposiciones del TUE que regulan el marco básico de actuación de la Unión Europea en materia de seguridad y defensa, y en segundo lugar, al incipiente desarrollo de una política europea de seguridad y defensa que fundamenta el establecimiento de una estructura que le permita realizar operaciones civiles y militares de gestión de crisis, no debe esconder las claras diferencias existentes entre los miembros de la Unión sobre el papel que esta última debe desempeñar en esta materia. Es decir, se han desarrollado los instrumentos necesarios sin haber trabajado seriamente en el establecimiento de una doctrina común que sirva para saber cuándo, dónde y para qué se utilizarán. Tal y como se ha apuntado con anterioridad, falta una concepción estratégica de la UE en este terreno ${ }^{20}$. En este sentido, la crisis del 11 de septiembre ${ }^{21}$ ha mostrado claramente la falta de cohesión política, visualizada en los intentos de establecer directorios o triunviratos, tal y como ocurrió con las reuniones restringidas de Gante entre los tres Grandes, antes de la celebración del Consejo Europeo, o de Downing Street, en las que se abordó el tema de las acciones militares contra Afganistán. Sin embargo, haciendo de la necesidad virtud, algunos analistas ya vinculan eficacia de la UE en el terreno de la gestión de crisis con mecanismos de directorio o de grupos reducidos ${ }^{22}$.

Conviene también señalar los problemas que se plantean con el actual diseño orgánico de la PESC y su coexistencia con la acción exterior de las Comunidades Europeas. Así, la coexistencia de diversos órganos con funciones conexas puede plantear problemas de coherencia que acaben dañando la credibilidad de la PESD. Este es un problema vital, que todo el mundo parece apreciar, y, de ahí, que sea necesario redefinir la estructura institucional que presenta la actual y difícil coexistencia en- 
tre la Presidencia de la Unión Europea, el Alto Representante de la PESC y el Comisario de Relaciones Exteriores.

Las ideas sobre el modelo a seguir son múltiples. Desde el Parlamento Europeo se ha propuesto integrar las funciones del Alto Representante en la Comisión, mientras que algunos expertos hacen propuestas bien diferentes. Así, por ejemplo, EVERTS ${ }^{23}$ propone abolir el papel de la presidencia en la PESC, mientras que DASSU y MISSIROLI proponen reforzar la posición del Alto representante de la PESC concediéndole la exclusiva representación de la Unión en materia PESC, así como el derecho de iniciativa y un aumento de los recursos económicos y materiales a su servicio. Asimismo, proponen que este «gran jefe» de la PESC presida el Consejo de ministros de Asuntos Exteriores y todo el sistema de la PESC, para lo cual estaría apoyado por dos adjuntos, el Comisario de Relaciones Exteriores y un representante itinerante de la PESC/PESD encargado del lado operacional de la gestión de crisis y de presidir el Comité Político y de Seguridad ${ }^{24}$. Lo que es evidente, en estos momentos, y aún más tras la presentación del informe de Javier Solana al Consejo Europeo de Barcelona sobre las reformas del funcionamiento del Consejo ${ }^{25}$, que afectaría al mecanismo de la presidencia, es que el diseño orgánico de la PESC tiene que cambiar.

Por otra parte, la implementación real y efectiva de la Política Europea de Seguridad y Defensa pasa ineludiblemente por la obtención de unos recursos y capacidades al servicio de la Unión que le sirva para desarrollar operaciones militares y civiles de gestión de crisis. Desde esta doble perspectiva se ha estado trabajando intensamente en los últimos años.

En el terreno militar, la Unión Europea ha elaborado un objetivo general y un objetivo de capacidades militares, para establecer un requisito pormenorizado que permita conocer las contribuciones nacionales necesarias para que la Unión Europea pueda dirigir operaciones militares. El catálogo de fuerzas está conformado por personas y por medios (aviones, barcos u otros) de catorce miembros de la UE, Dinamarca al margen, más de otros países europeos (Noruega) o de futuros miembros (Turquía, Hungría, República Checa, Eslovaquia, Chipre) ${ }^{26}$. Este trabajo en el marco de la Unión se completa con un mecanismo de evaluación que ha de garantizar el refuerzo europeo de capacidades, mediante un seguimiento e impulso de los avances en el cumplimiento de los compromisos asumidos con vista a lograr el objetivo global.

En el marco de la gestión civil de crisis, la Unión Europea ha elaborado dos auditorías: la primera sobre los recursos de la Unión y la segunda sobre los de los Estados miembros para poder así garantizar la co- 
ordinación entre ambos. El resultado final fue altamente satisfactorio al demostrar su preparación y experiencia para desarrollar un amplio abanico de operaciones. Aun así, el Consejo Europeo de Feira estableció el objetivo de pasar de los 3300 agentes policiales de los Estados miembros preparados para desplegarse en operaciones de la UE a poder suministrar 5000 policías para desarrollar misiones de toda la gama de operaciones de prevención y gestión de crisis. Además, se decidió que los Estados miembros debían comprometerse a determinar y desplegar en un plazo de treinta días fuerzas de policía que pudiesen realizar operaciones y misiones de asesoramiento, formación y supervisión policial, así como de policía operativa.

En tercer lugar, conviene señalar que la industria europea en materia de defensa se encuentra actualmente en una posición de clara desventaja respecto la norteamericana, que es más competitiva desde el punto de vista tecnológico y comercial. Aún más desde el 11 de septiembre. La decisión del presidente Bush de aumentar en un 14,5\% el presupuesto de defensa de cara al 2003 sitúa a los europeos en una posición de gran asimetría respecto a los Estados Unidos (en el año 2000, el presupuesto de defensa de los Estados Unidos equivalía a 1.029 euros por habitante frente a 323 euros por habitante en la Europa de los Quince). Es evidente que es necesario trabajar en esta materia si no se quiere depender tecnológicamente de EE.UU. El principal problema para avanzar en el terreno del armamento se encuentra en las dificultades que tiene la Unión Europea para actuar seriamente en la consecución de este objetivo.

En este sentido, algunas disposiciones del Tratado de la Comunidad Europea limitan la capacidad de acción de la Comunidad, desde su creación, en materias relacionadas con la defensa. Los artículos 296 a 298 del TCE establecen un régimen de reservas para la aplicación del tratado, justificado por exigencias de seguridad nacional ${ }^{27}$. El primero de estos preceptos autoriza a los Estados miembros a no aplicar las disposiciones del tratado a «la producción o el comercio de armas, municiones y material de guerra", siempre que sea necesario "para la protección de los intereses esenciales de su seguridad» $\mathrm{y}$ no alteren «las condiciones de competencia en el mercado común respecto de los productos que no estén destinados a fines específicamente militares». Este artículo se aplica únicamente a los productos recogidos en una lista aprobada por el Consejo el 15 de abril de 1958 que conforman el denominado hard defence material $^{28}$, que sólo puede tener fines militares, en contraposición al soft defence material ${ }^{29}$, que puede ser usado indistintamente con fines civiles o militares y que no estaría sujeto a esta reserva ${ }^{30}$. Por su parte, el artí- 
culo 297 TCE permite a los Estados miembros adoptar excepcionalmente medidas contrarias al Tratado o al derecho derivado en caso de graves disturbios internos, de guerra o de grave tensión internacional con amenaza de guerra, o de cumplimiento de las obligaciones contraídas para el mantenimiento de la paz y la seguridad internacional. Este régimen de reservas se completa con un sistema de control en su aplicación establecido por el artículo $298 \mathrm{TCE}$, en el que participa la Comisión y eventualmente el Tribùnal de Justicia.

Por otra parte, los compromisos adoptados por la Declaración adoptada en el Consejo Europeo de Colonia los días 3 y 4 de junio de 199 en el ámbito industrial y técnico de la defensa son menos concretos, puesto que los Estados miembros de la Unión Europea se declaran únicamente dispuestos a continuar los procesos existentes de reestructuración industrial y a avanzar, «según estimen adecuado los Estados miembros, en la armonización de los requisitos de carácter militar y en la planificación y adquisición de armas.» Esta aproximación a las cuestiones relativas a la industria y el mercado de la defensa está todavía muy lejos de las propuestas realizadas por la Comisión europea en dos comunicaciones sobre este tema presentadas en 1996 y $1997^{31}$.

Por su parte, algunos Estados miembros y ciertas empresas europeas han iniciado un proceso de acercamiento en materia de armamento, que completa precariamente los lentos avances que se han producido en el marco de la Unión Europea. En primer lugar, conviene recordar que los Estados miembros de la UEO cooperan en materia de armamento en el seno del GAEO (Groupe armement de l'Europe Occidentale) y de la OAEO (Organisation de l'armement de l'Europe Occidentale). Ambas instituciones han conseguido lanzar los programas EUCLID y THALES de investigación y tecnología, que han permitido la firma de contratos con diversas industrias europeas. Por su parte, la iniciativa franco-alemana, a la que se sumó Reino Unido e Italia, de crear el Organización Conjunta de Cooperación en materia de Armamento (OCCAR) también ha facilitado la cooperación en este ámbito. Su objetivo es «la gestión centralizada de grandes programas para el aprovechamiento de las economías de escala y el equilibrio en las relaciones entre suministradores y suministrados». En la actualidad, España, Países Bajos, Bélgica han solicitado su entrada a la organización y Suecia ya ha mostrado un gran interés por la iniciativa. Por otra parte, los ministros de defensa de Alemania, Reino Unido, España, Italia y Suecia firmaron en 1998 una Carta de intenciones para facilitar la reestructuración de sus industrias de defensa. Finalmente, destaca también la creación, a principios del año 2000, de la Compañía Europea de Aeronáutica, Defensa y Espacio 
La política europea de seguridad y defensa...

(EADS) cuyos socios fundadores eran DASA, AMS y CASA ${ }^{32}$. A pesar de que los avances en este terreno ya se habían visto afectados por decisiones nacionales -la decisión española de vender la empresa Santa Bárbara, fabricante del carro de combate Leopard a una empresa americana frente a la existencia de una oferta alemana; el anuncio alemán de reducir su presupuesto de defensa por motivos de déficit público- lo cierto es que el 11 de septiembre ha situado a los europeos frente a un hecho consumado: el despegue estadounidense en materia tecnológica derivado de la voluntad de ese país de poner en marcha programas de gran envergadura, como el programa antimisiles que supondrá una nueva era en materia tecnológica.

En suma, la situación no es nueva. Recuerda la situación de los primeros sesenta, en plena guerra fría, momento en el que Gran Bretaña, tuvo que optar por subirse al carro de la tecnología estadounidense (Polaris) o apostar por una inversión europea (franco-británica) en materia de armas nucleares. Hoy en día, el número de actores ha aumentado (ya son más de tres) y el sistema internacional se ha transformado, pero una cosa sigue igual en el terreno militar: la voluntad clara y decidida de Estados Unidos en materia armamentística frente a las dudas y a las diferencias de los europeos (dudas en torno a la eficiencia de la opción armamentística en el actual sistema internacional, temor a perder el tren de la carrera tecnológico-industrial, etc.). Es evidente que éste es un tema que combina concepciones diplomático-militares con intereses industriales. Todo ello genera un escenario mucho más complejo del que pueden abordar estas páginas ${ }^{33}$.

\section{Los desafíos externos}

La consecución de la Política Europea de Seguridad y Defensa está sujeta además a un conjunto de desafíos externos íntimamente relacionados a los que conviene dar respuesta a corto plazo. En este sentido, las relaciones con Estados Unidos, con los miembros europeos de la OTAN que no forman parte de la Unión Europea y con la OTAN son claves para el futuro de la Unión Europea en este ámbito.

La Administración, el Congreso y la opinión pública norteamericana, tras la finalización del periodo de guerra fría se han mostrado abiertamente a favor de que los europeos aumentasen su contribución a la seguridad del viejo continente ${ }^{34}$. Ello se debería implementar mediante el refuerzo del pilar europeo de la Alianza Atlántica, por lo que cualquier iniciativa europea no podía suponer un menoscabo del papel de la OTAN. 
Desde esta perspectiva, la Administración norteamericana ha apoyado el desarrollo de la política europea de seguridad y defensa siempre que se eviten las denominadas tres D's: decoupling, duplication and discrimination $^{35}$. Todo ello implicaba entre otras cosas el establecimiento de relaciones con la OTAN y la participación en la PESD de los aliados europeos que no sean miembros de la UE.

Sin embargo, las primeras decisiones adoptadas por la Unión Europea en relación con el refuerzo de la política europea de seguridad y defensa durante el Consejo Europeo de Helsinki, en diciembre de 1999, despertaron cierta desconfianza entre los norteamericanos. La formulación de dichas decisiones merece un análisis detallado, ya que en las mismas se basa la desconfianza estadounidense ${ }^{36}$.

En primer lugar, a diferencia de la UEO, cuyo desarrollo en los años noventa, había intentado dejar bien claro que se llevaba a cabo evitando duplicaciones con la OTAN, el Consejo Europeo afirmaba en Helsinki que la capacidad militar de la Unión Europea «evitará duplicaciones innecesarias». De ello se podría derivar, según se interprete el texto, que la UE podría producir duplicaciones con las capacidades norteamericanas si se considerará necesario para dotar a la Unión de una verdadera capacidad militar autónoma, independiente de ayudas externas. Es evidente que con esa formulación, la UE se está dejando una "puerta abierta». ¿Qué voluntad existe para cruzarla, política y presupuestariamente? Esa es otra cuestión.

En segundo lugar, los norteamericanos siempre han sido partidarios de que la OTAN sea la que tenga el derecho a ser la primera en rechazar la posibilidad de actuar en una crisis y que posteriormente sea la Unión Europea la que decida si realizará una determinada acción. En cambio, el Consejo Europeo de Helsinki, lejos de someterse explícitamente a esta condición, se limitó a afirmar su voluntad de desarrollar la capacidad de iniciar y llevar a cabo operaciones militares cuando «no participe la OTAN en su conjunto», sin realizar ninguna matización complementaria, por lo que también se ha creado una cierta polémica sobre el significado real de esta afirmación. Esta ambigüedad calculada de los europeos -muy propia de lenguajes de consenso entre quince voluntades políticas diferentes- abunda en el aspecto anterior. ¿Cuál es el escenario imaginable en el que los europeos actuarían, por encima de la voluntad contraria de los Estados Unidos?

En tercer lugar, las relaciones transatlánticas se enfrentan al problema tradicional del reparto de las cargas presupuestarias de la defensa (burden sharing). Este problema ha dificultado las relaciones entre los Estados Unidos y sus socios europeos de la OTAN desde los años sesen- 
La política europea de seguridad y defensa...

ta. Los norteamericanos pretenden que los europeos aumenten su presupuesto dedicado a la defensa (EE.UU. dedica un 2,8\% del PIB frente al $1,41 \%$ de la media de los países de la UE), asumiendo una parte importante de la mejora de capacidades necesarias para hacer frente a los desafíos militares que se le plantean a la OTAN, para lo cual se diseñó la Iniciativa de Capacidades de Defensa. Por su parte, los europeos han iniciado un proceso similar, pero desde la perspectiva de las necesidades que se le plantean a la Unión Europea para aumentar su capacidad militar de gestión de crisis. Todo ello ha provocado nuevas tensiones sobre lo que parece una duplicación de esfuerzos.

En cuarto lugar, otro foco de divergencias se encuentra en las prioridades establecidas para garantizar la seguridad. Así la iniciativa de defensa antimisiles defendida por EE.UU. con la llegada de la Administración Bush ha provocado el escepticismo de los europeos sobre si ése era el camino acertado, puesto que desde Europa se han alzado voces a favor de la utilización de otros instrumentos en la lucha contra las armas de destrucción masiva como los políticos, económicos y el control multilateral de armamento. El ejemplo más claro en este terreno, como veremos a continuación, son las diferencias que han aparecido entre la administración Bush y la Comisión, Patten en particular, en relación con el tratamiento a dar al Irak de Sadam Hussein. La política del «eje del mal» de la administración Bush choca de pleno con las preocupaciones existentes en la Comisión Europea en torno a los Estados en descomposición en el mundo, focos de conflictividad. Mientras Washington piensa en como destruir algunos Estados, Bruselas piensa en cómo evitar el fracaso de la lógica estatal en buena parte del mundo (Africa Subsahariana, ex glacis soviético).

Finalmente, llegamos, de modo concreto, a la situación creada tras los atentados terroristas del 11 de septiembre. Tras un primer momento en el que los europeos mostraron su solidaridad tanto individual como colectivamente en la OTAN con la activación del artículo 5 del TAN ${ }^{37}$, se ha entrado en una fase de discrepancia sobre el camino a seguir tras la finalización de la campaña militar en Afganistán. La voluntad de la Administración norteamericana de continuar la guerra contra el terrorismo con operaciones militares en otros puntos del planeta, empezando por Irak, ha contado con la crítica abierta de la Comisión Europea, la Presidencia española de la Unión Europea y de los gobiernos francés y alemán, que se muestran reticentes con el unilateralismo de EE.UU. En el fondo el problema que se plantea no es otro que el de la diferente percepción que se tiene en las dos orillas del Atlántico sobre como enfrentarse a las crisis internacionales. En efecto, tras la constitución de la 


\section{Esther Barbé, Alfonso González Bondia}

más lejos, el desarrollo de las estrategias comunes de la PESC ya se han visto enfrentadas al debate entre eficacia (vinculada a confidencialidad) y transparencia ${ }^{53}$.

Sobre la base de los objetivos genéricos de fomentar el establecimiento de unas consultas mutuas, una cooperación y una transparencia eficaces entre la OTAN y la Unión Europea, ambas instituciones internacionales han estado trabajando en la definición de un conjunto de objetivos específicos que satisfagan las necesidades concretas de cada una de ellas. Para ello, se ha trabajado sobre la base de la experiencia previa en las relaciones OTAN/UEO y de las nuevas exigencias de la Unión Europea, teniendo en cuenta las tareas que pretende desarrollar y los instrumentos con los que cuenta esta última. Desde esta perspectiva se han identificado hasta el momento los siguientes objetivos concretos en las relaciones OTAN/Unión Europea: el intercambio de información entre las dos instituciones internacionales, la realización de tareas de una organización en beneficio de la otra, la cesión de medios y la participación de una institución internacional en las actividades de la otra.

Sin embargo, a pesar de estos avances, conviene señalar que la consecución definitiva de unas relaciones estables entre la UE y la OTAN ha contado con un obstáculo que ha retrasado notablemente la adopción de acuerdos, como es la adopción de disposiciones satisfactorias para todos los miembros europeos de la OTAN que le permita su participación en las actividades en materia de seguridad y defensa de la Unión Europea. Desde esta perspectiva, la OTAN manifestó reiteradamente que nada se aprobaría hasta que todo estuviese aprobado ${ }^{54}$, por lo que la consecución de acuerdos definitivos sobre el intercambio de información, el acceso a las capacidades de planificación de la OTAN, la presunción de disponibilidad de los medios y capacidades de la OTAN para operaciones de la Unión Europea, la identificación de una cadena de mando europea en la estructura de la OTAN para operaciones de la Unión Europea y la adaptación del proceso de planificación de la defensa de la OTAN para incluir las necesidades de la Unión Europea, ha estado a la espera del cierre de un acuerdo satisfactorio sobre la participación de los aliados europeos en las actividades de la Unión Europea.

Así pues, en estos momentos, es tarea prioritaria de la presidencia española cerrar definitivamente dichos acuerdos, ya que esta cuestión está retrasando la que podría ser la primera operación militar de la Unión Europea: un despliegue de fuerzas en Macedonia, en sustitución de la OTAN, con el objetivo de proteger a los observadores internacionales del proceso de paz en dicho país, siempre y cuando la PESD, por si sola, esté lista para hacerse cargo de dicha operación. 
ciones dirigidas por la Unión Europea, una vez se haya decidido su inicio; aunque en el caso de que la operación se desarrolle con medios y capacidades de la OTAN, los miembros europeos de esta organización no pertenecientes a la Unión podrán solicitar libremente su participación sin que sea necesaria una invitación previa por parte del Consejo ${ }^{40}$. La implementación de las operaciones dirigidas por la Unión con fuerzas militares de terceros Estados requerirá de la creación de un Comité de contribuyentes encargado de la conducción diaria de la operación, a cuyas reuniones podrán asistir todos los miembros de la Unión y los terceros Estados participantes en la operación, aunque únicamente los Estados contribuyentes participarán en la gestión diaria. No obstante, conviene señalar que se mantiene la autonomía de la Unión, ya que la decisión sobre el final de la operación corresponderá únicamente al Comité Político y de Seguridad y al Consejo, que tendrán en cuenta los puntos de vista expresados por el Comité de contribuyentes ${ }^{41}$.

Sin embargo, Turquía no está satisfecha con las decisiones adoptadas por la Unión Europea en Feira y Niza al considerar que, como uno de los pilares de la seguridad en el interior de la OTAN, tiene algo que decir respecto a la PESD ${ }^{42}$. Por tanto, se opone a que la Unión Europea pueda acceder automáticamente al proceso de planificación de la OTAN y a la presunción de disponibilidad de sus medios y capacidades para la gestión de crisis, si la cuestión de su asociación a la PESD no se resuelve de manera satisfactoria para Turquía. Desde esta perspectiva, Turquía reclama estar asociada a todos los aspectos del proceso de reflexión y de decisión que lleven a la Unión Europea a intervenir en una crisis, aún respetando su derecho a decidir autónomamente. Por otra parte, el gobierno turco considera que la PESD puede comprometer el papel de la OTAN en materia de defensa. En este sentido, considera que una operación de la Unión podría degenerar en una misión del artículo 5 del TAN, por lo que todos los aliados deberían tener derecho a aceptar la utilización de los medios y capacidades de la OTAN por parte de la Unión. En la primavera del 2001, gracias a los esfuerzos diplomáticos del Reino Unido y EE.UU., se llegó a un acuerdo con el gobierno turco, que finalmente no llegó a ponerse en práctica como consecuencia del rechazo del Estado mayor general turco generado por la inquietud que provocaban el acceso garantizado de la Unión a las capacidades de planificación de la OTAN y los compromisos en materia de seguridad que se aplicaría a Chipre ${ }^{43}$. Asimismo, hay que apuntar que el gobierno griego mostró su malestar en relación con el método seguido para la negociación del mismo, mostrando su voluntad de rechazarlo en el marco de la UE. 
Las dificultades en lograr una solución satisfactoria para todas las partes han puesto, de nuevo, en alerta a Estados Unidos. En este sentido, el embajador norteamericano ante la OTAN alertó del peligro de que, si no se llega rápidamente a un acuerdo, en el seno de la Unión Europea, ésta se plantee la necesidad de dotarse de capacidades de planificación, sin recurrir a las de la OTAN y por lo tanto duplicándolas. Es evidente que la posibilidad existe, pero, ¿hasta qué punto los europeos están dispuestos a asumir sus consecuencias?. Esto es, el sentimiento de sentirse relegados tanto de Turquía como de Estados Unidos ${ }^{44}$.

El tercer desafío para la PESD viene determinado por sus relaciones con la OTAN. Durante el periodo en el que la UEO se erigió en el brazo armado de la Unión Europea, las relaciones de esta última con la OTAN eran prácticamente testimoniales, limitándose a la celebración de reuniones informales de trabajo en las que participaban representantes de ambas instituciones para tratar temas de interés común. Esta situación empezó a cambiar substancialmente a partir del momento en el que la Unión Europea decidió desarrollar su capacidad operativa para la gestión militar de crisis.

En la Cumbre de la OTAN, celebrada en Washington en 1999 tras la campaña militar contra Serbia, los aliados mostraron su satisfacción con la decisión de la Unión Europea de fortalecer la política europea común en materia de seguridad y defensa y acordaron «definir y adoptar las disposiciones necesarias para que la Unión Europea pueda acceder fácilmente a los medios y capacidades colectivos de la Alianza para llevar a cabo operaciones en que la OTAN como conjunto no se vea implicada militarmente como Alianza" ${ }^{45}$. En este mismo sentido, el Concepto Estratégico de la Alianza, adoptado también en esa reunión de la OTAN, contemplaba la compatibilidad entre la OTAN y la política de defensa común a desarrollar en el seno de la UE, tal y como se recoge en el Tratado de Amsterdam, y reconocía la necesidad de una cooperación estrecha entre ambas instituciones para construir una identidad europea de seguridad y defensa en el seno de la OTAN con el objeto, entre otras cosas, de «ayudar a los aliados europeos a actuar por si mismos según convenga mediante la disposición de la Alianza a poner sus medios y capacidades, caso por caso y por consenso, a disposición de operaciones en las que la Alianza no se vea implicada militarmente (...), teniendo en cuenta la plena participación de todos los Aliados europeos si así lo decidiesen.» ${ }^{46 .}$

Desde esta perspectiva, ambas instituciones decidieron el establecimiento de consultas mutuas, de relaciones de cooperación y de transparencia entre ellas; para lo cual se empezó a trabajar en el establecimiento de mecanismos que facilitarían el cumplimiento de dichos objetivos ${ }^{47}$. 
Para ello, se adaptarían las relaciones preexistentes entre la OTAN y la UEO, teniendo en cuenta la distinta naturaleza de la OTAN y la Unión Europea. Así, se acordó que las relaciones se materializarían en un primer momento a través de contactos informales entre el Secretario General del Consejo de la Unión Europea y el Secretario General de la OTAN.

Algunos de estos objetivos se concretaron durante el primer semestre de 2000, cuando en el marco del desarrollo de los objetivos generales y de las capacidades colectivas que ha de permitir a la Unión Europea la realización de operaciones militares, esta última decidió solicitar asesoramiento técnico a la OTAN ${ }^{48}$. Por su parte, la Unión europea acordó en la reunión del Consejo Europeo celebrada en Santa Maria de Feira el 19 y 20 de junio de 2000, los principios que deberían regir sus relaciones con la OTAN, proponiendo a esta última una agenda de temas y modalidades de trabajo durante un periodo provisional. Así, se decidió proponer a la OTAN la creación de cuatro grupos de trabajo ad hoc compuestos por representantes de ambas instituciones que empezarían a trabajar conjuntamente en la definición de objetivos de capacidad, disposiciones para permitir a la UE acceder a los medios y capacidades de la OTAN y la definición del régimen permanente que regulará las relaciones entre ambas instituciones ${ }^{49}$. Esta propuesta fue seguidamente implementada, por lo que los trabajos en estas materias avanzaron rápidamente.

En la reunión del Consejo Europeo celebrada en Niza, la Unión Europea acordó que la consulta y cooperación entre ambas instituciones se desarrollaría en cuestiones de seguridad, defensa y gestión de crisis, de interés común, con vistas a permitir la respuesta militar más apropiada a una crisis y garantizar una gestión de crisis eficaz» ${ }^{50}$. Lo que para la OTAN suponía el establecimiento de «una verdadera asociación estratégica en la gestión de crisis entre la OTAN y la Unión Europea» ${ }^{51}$. Unos pocos días más tarde, ambas instituciones acordaron un dispositivo permanente de consulta y cooperación consistente en la celebración, durante cada presidencia de la Unión Europea, de no menos de tres reuniones del Consejo del Atlántico Norte con el Comité Político y de Seguridad, y al menos una reunión ministerial UE-OTAN ${ }^{52}$. Otro ejemplo de avances en las relaciones entre la OTAN y la UEO se encuentra en el acuerdo provisional sobre la seguridad de la información firmado entre ambas instituciones, para facilitar la transparencia mediante el intercambio de información. Es evidente que estas cuestiones plantean dificultades en el marco de la UE, donde algunos países luchan por crear una cultura de transparencia. El carácter secreto que comporta la puesta en marcha de una defensa común choca, sin dủda, con la voluntad de algunos países de hacer más transparente todo el proceso de construcción europea. Sin ir 
más lejos, el desarrollo de las estrategias comunes de la PESC ya se han visto enfrentadas al debate entre eficacia (vinculada a confidencialidad) y transparencia ${ }^{53}$.

Sobre la base de los objetivos genéricos de fomentar el establecimiento de unas consultas mutuas, una cooperación y una transparencia eficaces entre la OTAN y la Unión Europea, ambas instituciones internacionales han estado trabajando en la definición de un conjunto de objetivos específicos que satisfagan las necesidades concretas de cada una de ellas. Para ello, se ha trabajado sobre la base de la experiencia previa en las relaciones OTAN/UEO y de las nuevas exigencias de la Unión Europea, teniendo en cuenta las tareas que pretende desarrollar y los instrumentos con los que cuenta esta última. Desde esta perspectiva se han identificado hasta el momento los siguientes objetivos concretos en las relaciones OTAN/Unión Europea: el intercambio de información entre las dos instituciones internacionales, la realización de tareas de una organización en beneficio de la otra, la cesión de medios y la participación de una institución internacional en las actividades de la otra.

Sin embargo, a pesar de estos avances, conviene señalar que la consecución definitiva de unas relaciones estables entre la UE y la OTAN ha contado con un obstáculo que ha retrasado notablemente la adopción de acuerdos, como es la adopción de disposiciones satisfactorias para todos los miembros europeos de la OTAN que le permita su participación en las actividades en materia de seguridad y defensa de la Unión Europea. Desde esta perspectiva, la OTAN manifestó reiteradamente que nada se aprobaría hasta que todo estuviese aprobado ${ }^{54}$, por lo que la consecución de acuerdos definitivos sobre el intercambio de información, el acceso a las capacidades de planificación de la OTAN, la presunción de disponibilidad de los medios y capacidades de la OTAN para operaciones de la Unión Europea, la identificación de una cadena de mando europea en la estructura de la OTAN para operaciones de la Unión Europea y la adaptación del proceso de planificación de la defensa de la OTAN para incluir las necesidades de la Unión Europea, ha estado a la espera del cierre de un acuerdo satisfactorio sobre la participación de los aliados europeos en las actividades de la Unión Europea.

Así pues, en estos momentos, es tarea prioritaria de la presidencia española cerrar definitivamente dichos acuerdos, ya que esta cuestión está retrasando la que podría ser la primera operación militar de la Unión Europea: un despliegue de fuerzas en Macedonia, en sustitución de la OTAN, con el objetivo de proteger a los observadores internacionales del proceso de paz en dicho país, siempre y cuando la PESD, por si sola, esté lista para hacerse cargo de dicha operación. 


\section{Consideraciones finales}

Ante la posibilidad de desplegar fuerzas militares de la UE en Macedonia para sustituir a la OTAN -tema discutido en un encuentro informal del Consejo, en Cáceres, bajo presidencia española- el Secretario de Defensa británico, Geoff Hoon, simplemente apuntó que existe «un riesgo muy alto de fracaso» ${ }^{55}$, ante las deficiencias de la UE en áreas vitales para la acción militar (transporte aéreo, logística y comunicaciones).

De dichas deficiencias técnicas se deriva una constatación política: la dependencia de los europeos con respecto a la OTAN. Lo que sitúa el papel de la UE en el mundo en materia de gestión militar de crisis, si se quiere poner en estos términos, en manos de Washington y/o de Ankara. Es cierto, pero excesivo.

No permitamos que los árboles no nos dejen ver el bosque. Antes de concluir que el papel de la UE en el mundo en materia de gestión militar de crisis se ve imposibilitado por sus carencias técnicas y por la falta de acuerdo UE/OTAN, hay que recordar una serie de deficiencias internas, ajenas a los imponderables externos. Así, se puede hablar de la falta de un concepto estratégico basado en unas prioridades claramente definidas por la UE y necesario para cualquier política de defensa (¿porqué Macedonia?), de la falta de sinergias e incluso incoherencias entre pilares o entre la Unión y sus Estados miembros (¿porqué no vincular claramente la ayuda al desarrollo con los objetivos de prevención de conflictos o de gestión de crisis de la PESC/PESD?) o de los problemas institucionales derivados de una falta de confianza en el desarrollo de la PESC que ha llevado al establecimiento de una triple representación exterior de la Unión Europea personificada en el Comisario de Relaciones Exteriores, el Alto Representante de la PESC y la Presidencia (¿porqué no se simplifica la representación exterior y se le dota de poderes específicos para ejercer un claro liderazgo que dé credibilidad a la Unión?). Es más, conectando con el punto de partida de estas páginas, no está de más preguntarse hasta qué punto están convencidos los europeos de la necesidad de contar con instrumentos militares para defender su papel en el mundo. Ahí se halla el tema central del debate: primero, preguntarnos qué queremos hacer y, segundo, cómo hacerlo. La PESD, por tanto, viene en segundo lugar. Primero, hay que ver el bosque.

\section{Notas}

1 El tema se plantea en el momento actual, muy vinculado, a los acontecimientos de los últimos años que han situado a los europeos frente a la opción de «actuar» con medios 
militares (Bosnia, Kosovo, Afganistán). Sin embargo, el debate en torno a sí la voluntad de la CE de convertirse en potencia mundial era posible sin instrumentos militares viene de lejos. Véase, en ese sentido, el texto de BULL, Hedley (1983): "Civilian power Europea: A contradicition in terms?». En TSOUKALIS, L.: The European Community: Past. Present and Future, Oxford, Blackwell.

2 El tema ha generado una literatura numerosa en nuestro país. Para una visión introductoria al mismo, véase GONZÁLEZ BONDIA, Alfonso (2000): «La política de defensa de la Unión Europea». En BARBÉ, Esther (coord.): Política Exterior Europea, Barcelona, Ariel, pp. 129-157. Para la situación post-Niza, véase, entre otros, GONZÁLEZ ALONSO, Luis N. (2001): "La política europea de seguridad y defensa después de Niza", Revista de Derecho Comunitario Europeo, núm. 9, enero-junio, pp. 197-238; URBINA, Julio Jorge (2001): "Reflexiones en torno a la configuración de una política de seguridad y defensa en el seno de la Unión Europea", Revista de Derecho Comunitario Europeo, núm. 10, julio-diciembre, pp. 439-471; SÁNCHEZ RAMOS, Belen (2001): «La Política Exterior y de Seguridad Común de la Unión Europea tras el Tratado de Niza", Anuairo da Facultade de Dereito da Universidade da Coruña, núm. 5, pp. 821-834; GONZÁLEZ VEGA, Javier A. (2001): «Los acuerdos de Niza, la PESC y la arquitectura europea de seguridad y defensa", Boletín Europeo de la Universidad de La Rioja, suplemento, núm. 9, diciembre, pp. 11-28; MARRERO ROCHA, Inmaculada C. (2001): «Avances en el ámbito de la seguridad y la defensa europea", Tiempo de Paz, núm. 62, otoño.

3 Se trata de las conocidas como misiones Petersberg, ya que fueron definidas por primera vez en la sesión ministerial del Consejo de la UEO que se celebró en el Castillo de Petersberg, cerca de Bonn, el 19 de junio de 1992. Así, en virtud de la Declaración de Petersberg, la UEO identificó un conjunto de misiones tipo que servirían de referencia para definir su desarrollo operativo. Sobre este tema véase GRASSI, S. (1998): "L'introduzzione delle operazioni di peace-keeping nel Trattato di Amsterdam: profili giuridici ed implicazioni politiche», La Comunità Internazionale, vol. LIII, núm. 2, pp. 295-326.

${ }^{4} \mathrm{La}$ introducción de las misiones Petersberg en Amsterdam se explica, en buena medida, por el deseo de los nuevos miembros de la UE, fundamentalmente Suecia y Finlandia, de definir un marco conceptual acorde con sus propias políticas de seguridad. Se puede apuntar, de modo eufemístico, que la reforma de Amsterdam acercó la PESC a la sensibilidad nórdica en materia internacional. Véase, en ese sentido, BARBÉ, E. y JOHANSSON, E. (2001): «De Niza a Göteborg. La UE en manos nórdicas», Política Exterior, vol. XV, núm. 80 , marzo/abril, pp. 21-28. Al mismo tiempo, esta clarificación alejaba los objetivos expresados por otros Estados miembros, especialmente Grecia, de plantear la PESD como una política de defensa colectiva de los Quince.

5 UE, Parlamento Europeo, Informe sobre la elaboración de una política europea común en materia de seguridad y defensa después de Colonia y Helsinki - Comisión de Asuntos Exteriores, Derechos Humanos, Seguridad Común y Política de Defensa, Ponente: Catherine LALUMIERE, A5-0339/2000, pp.27-28.

${ }^{6} \mathrm{El}$ artículo 23.2 in fine del TUE ha previsto que les decisiones que repercutan en el ámbito militar o de la defensa no se podrán adoptar por mayoría cualificada. Por su parte, la reforma de Niza ha introducido el mecanismo de la cooperación reforzada en el segundo pilar, pero dejando al margen «las cuestiones que tengan repercusiones militares o repercusiones en el ámbito de la defensa» (art. $27 \mathrm{~B}$ ).

7 En este sentido, véase el ejemplo ofrecido en materia de cooperación franco-británica por VAN HAM, P. (2000): «Franco British Defence Cooperation and Compatibility of 


\section{La política europea de seguridad y defensa...}

ESDP with NATO». En DUKE, Simon: Between Vision and Reality. CFSP's Progress on the Path to Maturity, Maastricht, European Institute of Public Administration.

8 Para un conocimiento más extenso de esta iniciativa, véase GUILLAUME, M. (1998): "L'organisation conjointe de coopération en matière d'armement", Annuaire Français de Droit International, vol. XLIV, pp. 283-297.

${ }^{9}$ La fractura entre atlantistas y europeístas recubría una doble dimensión de la política de los estados europeos (su visión de la defensa europea y, a través de ella, el mayor o menor papel a desempeñar por los Estados Unidos en la misma). Sobre el particular, véase BARBE, Esther (1995): La seguridad en la nueva Europa, Madrid, Libros de la Catarata. Sin que se puede hablar hoy en día de la completa desaparición de tal fractura, sí que se puede afirmar que la misma se ha mitigado tras el fin de la guerra fría, dándose lugar a situaciones (como el conflicto de Kosovo) en las que la posición británica está más cercana a la francesa que a la de los Estados Unidos.

10 Véase, en ese sentido, la comunicación de la Comisión sobre prevención de conflictos [COM (2001) 211 final].

11 Véase sobre todo la literatura académica generada por el Conflict Prevention Network, auspiciado por la UE y dirigido por Reinhardt Rummel.

12 Sobre el funcionamiento de los órganos de gestión de crisis de la UE a partir de los ataques del 11 de setiembre, véase MIRALLES, Débora (2001): Actuació de la troüka després dels atacs de l'11 de setembre (working paper, 10/2001) en la sección «Especial 11 de septiembre" del Observatorio de Política Exterior Europea (IUEE), http://www.uab.es/iuee.

13 El hecho de que un general finlandés se halle al frente del Estado Mayor nos ayuda a «relativizar» la poca importancia que habitualmente se atribuye a los socios neutrales en el marco de la PESD.

14 Voces autorizadas han destacado el hecho de que las funciones de alerta temprana que debería realizar dicho órgano se ven mermadas por sus escasos recursos y por su función básica hasta el momento, haberse convertido en una extensión del gabinete del Alto Representante PESC. Véase $E U$ crisis response capability, International Crisis Group Issues Report n. 2, 26 Junio 2001, http://www.crisisweb.org/projects/issues/eu/reports.

${ }_{15} \mathrm{La}$ falta de estrategia de la UE en este terreno es destacada, entre otros, por HILL, Christopher (2001): "The EU's Capacity for Conflict Prevention», European Foreign Affairs Review, núm 6, pp. Pp. 315-333 o por PÉREZ CALDENTEY, Ignacio (2001): La necesidad de un concepto estratégico europeo (working paper, 12/2001) y BARBE, Esther y JOHANSSON, Elisabeth (2001): EU and Conflict Prevention (working paper 8/2001) en Observatorio de Política Exterior Europea, hppt://www.uab.es/iuee.

16 Reglamento (CE) núm. 381/2001 del Consejo de 26 de febrero por el que se crea un mecanismo de reacción rápida, DOCE, serie L, núm. 57, pp. 5-9.

17 La existencia de intereses nacionales diferentes ha sido una de las dificultades básicas de la construcción de una política europea y de seguridad y defensa. Véase, en ese sentido, BARBÉ, Esther (1997): «European Values and National Interests». En LANDAU, Alice y WHITMAN, Richard: Rethinkint the European Union. Institutions, Interests and Identities, Londres, Macmillan, pp. 129-146.

18 Consejo Europeo, «Conclusiones de la Presidencia, Parte B. Dinamarca y el Tratado de la Unión Europea, Anexo I. Decisión de los jefes de Estado y de Gobierno, reunidos en el seno del Consejo Europeo, relativa a determinados problemas planteados por Dinamarca en relación con el Tratado de la Unión Europea», DOCE, serie C, núm. 348, 21-12-1992. 
19 La dimisión del Ministro de Asuntos Exteriores, tras el Consejo Europeo de Niza fue ilustrativa en su momento. Sobre el tema, véase, LAURSEN, Finn (2001): «Denmark and the Treaty of Nice", paper presentado en 4th Pan-European International Relations Conference, Canterbury, 6-10 setiembre 2001.

20 Desde esta perspectiva, el Grupo de reflexión sobre la Presidencia española de 2002 del Instituto de Estudios Europeos de la Universidad San Pablo CEU considera necesaria la elaboración de un Concepto Estratégico de la Unión Europea que aclare diversos aspectos de la PESD como, por ejemplo, los objetivos, presupuestos y su área geográfica de actuación. Grupo de reflexión sobre la Presidencia española de 2002 (2001): La Política Europea Común de Seguridad y Defensa ante la Presidencia Española, Instituto de Estudios Europeos de la Universidad San Pablo CEU, Madrid, noviembre, pp. 37-38.

21 Para una reflexión en torno a la crisis del 11 de septiembre y sus efectos sobre la Política Exterior Europea, véase el apartado «11 de septiembre» de Working Papers en el Observatorio de Política Exterior Europea (IUEE), hppt://www.uab.es/iuee.

22 En este sentido, véase KEUKELEIRE, Stephan (2001): "Directorates in the CFSP/CESDP of the European Union: A plea for «Restricted Crisis Management Groups", European Foreign Affairs Review, núm. 6, pp. 75-101.

23 EVERTS, Steven (2002): Shaping a Credible EU Foreign Policy, Londres, Centre for European Reform.

24 DASSU, M. y MISSIROLI, A. (2002): Las estructuras de la Política Exterior y de Seguridad Común, hppt://www.ue2002.es/debate.

25 UE, Secretario General del Consejo de la Unión Europea, Preparing the Council for the enlargement, S0044/02, Bruselas, 11/3/2002.

${ }^{26}$ UEO, Asamblea, Mise en place des moyens et des capacités de gestion de crise pour la PECSD - Réponse au rapport annuel du Conseil. Rapport presenté au nom de la Comision de Défense par M. Rapon, (Document C/1715), 14 de noviembre de 2000.

27 Sobre el alcance de estos preceptos, véase GILSDORF, P. (1994): «Les réserves de sécurité du Traité CEE, à la lumière du Traité sur l'Union Européenne", Revue du Marché commun et de l'Union européenne, núm. 374, enero, pp. 17-25.

28 Esta compuesto por tanques, aviones de combate, barcos de guerra, etc.

29 Como, por ejemplo, vehículos todoterreno, barcos de rescate, aviones de transporte, etc.

30 Véase TRYBUS, M. (1998): «European Defence Procurement: Towards a Comprehensive Approach", European Public Law, vol. 4, núm. 1, marzo, pág. 113.

31 UE, Comisión Europea, Comunicación de la Comisión. Problemas de la industria europea relacionada con la defensa: propuestas de actuación a nivel europeo, COM(96) 10 final, Bruselas, 24-1-1996 y UE, Comisión Europea, Comunicación de la Comisión al Consejo, al Parlamento Europeo, al Comité Económico y Social y al Comité de las Regiones. La aplicación de la estrategia de la Unión sobre los sectores industriales vinculados a la defensa, COM (97) 583 final, Bruselas, 4-12-1997.

32 MEZZADRI, Sandra (2000): «L'oeuverture des marches de défense: enjeux et modalités", Publication Occasionnelle núm. 12, Instituto de Estudios de la Seguridad (UEO).

33 Para tener una idea más amplia sobre el tema, véase HEISBOURG, François (coord.): Défense européenne: la mise en oeuvre (Cahiers de Chaillot, núm. 42, septiembre).

34 Una visión actual del tema en GÓMEZ GARRIDO, Mercedes (2002): The European Security and Defense Policy (ESDP): The Recent Debate in the United States (working paper18/2002), disponible en Observatorio de Política Exterior Europea (IUEE), hppt://www.uab.es/iuee. 


\section{La política europea de seguridad y defensa...}

35 Estas condiciones fueron enunciadas por primera vez por la Secretaria de Estado norteamericana Madeleine Albright en el artículo titulado "The right balance will secure NATO's future" publicado en el Financial Times el 8 de diciembre de 1998.

36 Sobre los problemas de comprensión en Estados Unidos de esta iniciativa de la Unión Europea es ilustrativo el informe de las reuniones del Grupo sobre la Identidad Europea de Seguridad y Defensa de la Asamblea Parlamentaria del Atlántico Norte que se emitió en enero del 2000. Véase NATO Parliamentary Assembly, ESDI Steering Group, Summary Overview of the Discussions of the ESDI Steering Group Meetings, Madrid, 17-12-1999, Secretariat Report AT10ESDI(00)2, issued January 2000, hppt://www.naa.be/publications/special/at 10esdi002.html.

37 Más detalles sobre el tema en GONZÁLEZ BONDIA, Alfonso (2001): La OTAN y la crisis del 11 de septiembre (working paper 13/2001) Especial 11 de septiembre en Observatorio de Política Exterior Europea (IUEE), hppt://www.uab.es/iuee.

38 UE, Consejo Europeo, Conclusiones de la Presidencia, PESD: Informe de la Presidencia, Anexo VI-Dispositivos relativos a los Estados europeos miembros de la OTAN no pertenecientes a la UE y otros países que son candidatos a la adhesión a la UE, Niza, 9/12/2000, apartado II.

39 UE, Consejo Europeo, Conclusiones de la Presidencia, PESD: Informe de la Presidencia, Niza, 9/12/2000, apartado VI.

40 Sobre la problemática derivada de la participación de los Estados europeos de la OTAN que no son miembros de la Unión en la operaciones desarrolladas en el marco la PESD, véase CEBECI, M. (1999): A delicate process of participation: The question of participation of WEU Associate Members in decision-making for EU-led Petersberg operations, with special reference to Turkey, Institute for Security Studies, WEU, Occasional Papers 10, París, noviembre.

41 UE, Consejo Europeo, Conclusiones de la Presidencia, PESD: Informe de la Presidencia, Anexo VI - Dispositivos relativos a los Estados europeos miembros de la OTAN no pertenecientes a la UE y otros países que son candidatos a la adhesión a la UE, Niza, 9/12/2000, apartado III.

42 Sobre la posición de Turquía, véase ORHUN, Ö. (2000): «European Security and Defence Identity - Common European Security and Defence Policy", Perceptions. Journal of international affairs, septiembre-noviembre, vol. V, núm. 3, hppt://www.mfa.gov.tr/ grupa/percept/V-3/oorhum-9.htm.

43 Para una visión más amplia de los acontecimientos, véase VAN EEKELEN, W. (2001): L'OTAN et la politique européenne de sécurité et de défense, NATO Parlamentary Assembly, Rapport No. AU 200 DSC/TC (01) 4 rev. 1, octubre 2001, puntos 47 a 57.

44 VERSHBOW, A. (2000): "The American Perspective on ESDI/ESDP», Perceptions. Journal of international affairs, septiembre-noviembre, vol. V, núm. 3, disponible en internet, hppt://www.mfa.gov.tr/grupa/percept/V-3/avershbow-7.htm.

45 OTAN, Consejo del Atlántico Norte, Una Alianza para el siglo XXI, Washington, 24/04/1999, puntos 9 y 10.

46 OTAN, Consejo del Atlántico Norte, Concepto Estratégico de la Alianza, Washington, $23-24 / 4 / 1999$, punto 30 .

47 OTAN, Consejo del Atlántico Norte, Una Alianza para el siglo XXI, Washington, 24/04/1999, punto 9. b. y UE, Consejo Europeo, Conclusiones de la Presidencia, Anexo III - Declaración del Consejo Europeo sobre el refuerzo de la Política Europea Común de Seguridad y de Defensa, Colonia, 4/6/1999, punto 3. 
48 UE, Consejo Europeo, Conclusiones de la Presidencia, Santa Maria de Feira, 20/6/2000, punto 8. Posteriormente la OTAN se mostró dispuesta a proporcionar, sujeta a las decisiones necesarias, ulterior asesoría de expertos a petición de la UE. OTAN, Consejo del Atlántico Norte, Communiqué final, Bruselas, 15/12/2000, punto 30.

49 UE, Consejo Europeo, Conclusiones de la Presidencia, Apéndice 2 - Principios que deberán regir las consultas con la OTAN en materia militar y recomendaciones para desarrollar las formas que deberán revestir las relaciones entre la Unión Europea y la OTAN, Santa Maria de Feira, 20/6/2000.

50 UE, Consejo Europeo, Conclusiones de la Presidencia, Informe de la Presidencia sobre la Politica Europea de Seguridad y defensa, Niza, 7-8/12/2000.

51 OTAN, Consejo del Atlántico Norte, Communiqué final, Bruselas, 15/12/2000, punto 28.

52 Acuerdo formalizado en las resoluciones adoptadas en OTAN, Consejo del Atlántico Norte, Communiqué final, Bruselas, 14-15/12/2001, punto 31 y UE, Consejo, Comunicado de Prensa, Bruselas, 22-23/1/2001. Ambas decisiones dieron lugar a un intercambio de cartas, en enero del 2001, entre el Secretario General de la OTAN y la Presidencia de la Unión Europea, tal y como se referencia en OTAN, Consejo del Atlántico Norte, Communiqué final, Budapest, 29/5/2001, punto 42.

53 Sobre la necesidad de modificar las estrategias comunes para darles carácter reservado, véase el Informe del Alto Representante/Secretario General (Consejo de la Unión Europea, 14871/00, Bruselas 21 de diciembre de 2000, desclasificado el 30 de enero de 2001).

${ }^{54}$ OTAN, Consejo del Atlántico Norte, Communiqué final, Budapest, 29/5/2001, punto 49 .

55 Agence Europe, núm. 8163, 4 de marzo de 2002. 